\title{
The North/South Ireland Food Consumption Survey: vitamin intakes in 18-64-year-old adults
}

\author{
MM O'Brien ${ }^{1, *}, M_{\text {Kiely }}{ }^{1}$, KE Harrington ${ }^{2}$, PJ Robson ${ }^{3}$, JJ Strain ${ }^{3}$ and A Flynn ${ }^{1}$
}

Irish Universities Nutrition Alliance (IUNA) at: 'Nutritional Sciences, Department of Food Science, Food Technology and Nutrition, University College, Cork, Republic of Ireland: ${ }^{2}$ Department of Clinical Medicine, Trinity Centre for Health Sciences, St. James's Hospital, Dublin 8, Republic of Ireland: ${ }^{3}$ Northern Ireland Centre for Diet and Health (NICHE), University of Ulster, Coleraine, Co. Londonderry, BT52 1SA, Northern Ireland

\begin{abstract}
Objective: To estimate vitamin intakes and assess the contribution of different food groups to vitamin intakes in adults aged 18-64 years in Ireland as estimated in the North/South Ireland Food Consumption Survey. Intakes are reported for retinol, carotene, total vitamin $\mathrm{A}$, vitamin $\mathrm{D}$, vitamin $\mathrm{E}$, thiamin, riboflavin, pre-formed niacin, total niacin equivalents, vitamin $\mathrm{B}_{6}$, vitamin $\mathrm{B}_{12}$, folate, biotin, pantothenate and vitamin $C$. The adequacy of vitamin intakes in the population and the risk of occurrence of excessive vitamin intakes are also assessed.

Design: Food consumption was estimated using a 7-day food diary for a representative sample $(n=1379 ; 662 \mathrm{men}$ and 717 women) of 18-64-year-old adults in the Republic of Ireland and Northern Ireland selected randomly from the electoral register. Vitamin intakes were estimated using tables of food composition. Results: In general, the percentage of the population with vitamin intakes below the average requirement (AR) was low. Mean daily intake of total vitamin A was below the AR in $20.2 \%$ and $16.6 \%$ of men and women, respectively, and mean daily intake of riboflavin was below the AR in $12.5 \%$ and $20.6 \%$ of men and women, respectively. Mean daily folate intakes were below the AR for folate in $11.2 \%$ and $6.6 \%$ of women aged $18-35$ years and $36-50$ years, respectively. Only $2.2 \%$ of women aged $18-35$ years and $5.2 \%$ of women aged $36-50$ years achieved the recommended folate intake of $600 \mu \mathrm{g} \mathrm{day}^{-1}$ for women of reproductive age for the prevention of neural tube defects. A high proportion of the population has a low dietary vitamin D intake and is largely dependent on sunlight exposure to maintain adequate vitamin D status. Except for pre-formed niacin, the 95th percentile intake of vitamins did not exceed the tolerable upper intake level (UL) for any group and was much less than the UL for most vitamins. Although $20.8 \%$ of men and $6.3 \%$ of women exceeded the UL for pre-formed niacin (which is $35 \mathrm{mg}$, based on nicotinic-acid-induced flushing), the large contribution of meat and fish to the intake of niacin (as nicotinamide) suggests that the risk of overexposure to nicotinic acid is much lower than this and is probably solely related to supplement use. A small proportion of men (4.0\%) and women (1.2\%) aged 51-64 years had retinol intakes that exceeded the UL $(3000 \mu \mathrm{g})$ and while the 95th percentile intake of women in the 18-50 year age group was well below the UL, $1.5 \%$ of $18-35$-year-old and $2.4 \%$ of 36-50-year-old women had mean daily retinol intakes above the UL. About $2.0 \%$ of women had intakes of vitamin $\mathrm{B}_{6}$ that exceeded the UL $(25 \mathrm{mg})$. There were significant differences by age and sex in nutrient densities of vitamin intakes between men and women and between age groups, which may be explained by differences in consumption of particular food groups as well as different patterns of supplement use.

Conclusion: Nutritional adequacy of the population for most vitamins was good. Folate intake in women of childbearing age is not meeting current recommendations for the prevention of neural tube defects. The public health significance of the relatively high proportion of men and women with inadequate intakes of vitamin A and riboflavin and with low dietary intakes of vitamin $D$ is unclear and should be investigated further. With the possible exception of niacin (flushing) and vitamin $\mathrm{B}_{6}$ (neuropathy), there appears to be little risk of the occurrence of adverse effects due to excessive consumption of vitamins in this population, based on current dietary practices.
\end{abstract}


Recommended daily intakes have been established for vitamins by different authorities throughout Europe including the Republic of Ireland ${ }^{1}$, the United Kingdom ${ }^{2}$ and the European Union $(\mathrm{EU})^{3}$. In addition, a specific recommendation for folate has been made for women of reproductive age to help prevent neural tube defects ${ }^{4,5}$. Although for some vitamins evidence exists of health benefits of vitamin intakes above the currently recommended daily intakes, e.g. the reduction of plasma homocysteine and the risk of coronary heart disease by folate ${ }^{6}$ or the reduction of the risk of certain cancers by vitamin $C^{7}$, there is no consensus as of yet on these issues.

The increased use of nutritional supplements and the wider consumption of fortified foods in a number of countries have focused attention on the possible risks of excessive intakes of vitamins. Tolerable upper levels of intake (ULs) have been established for most vitamins by the Food and Nutrition Board based on the available evidence of adverse effects ${ }^{8-11}$. Recently, tolerable upper levels of intake (ULs) have been established by the $\mathrm{EU}$ for $\beta$-carotene ${ }^{12}$, riboflavin ${ }^{13}$, vitamin $\mathrm{B}_{6}{ }^{14}$, vitamin $\mathrm{B}_{12}{ }^{15}$ and folate ${ }^{16}$.

Vitamin intakes have been estimated in representative surveys of populations in a number of European countries $^{17,18}$. There is little information on vitamin intake available for Ireland, however, with the most recent information on vitamin intakes coming from the Irish National Nutrition Survey ${ }^{19}$ in the Republic of Ireland, published 10 years ago.

The North/South Ireland Food Consumption Survey was carried out to establish a database of habitual food and drink consumption in a representative sample of adults aged 18-64 years in Ireland. In this paper mean daily vitamin intakes and the major foods contributing to these intakes are reported. The adequacy of vitamin intakes in the population and the risk of the occurrence of excessive intakes are also assessed.

\section{Methods}

Detailed accounts of the methodologies ${ }^{20}$ and sampling procedure $^{21}$ used in this survey are provided in accompanying papers. From 1997 to 1999, the North/South Ireland Food Consumption Survey collected food intake data in a representative sample of 18-64-year-old adults ( $n=1379 ; 662$ men and 717 women) in the Republic of Ireland and Northern Ireland who were randomly selected from the electoral register. Pregnant/lactating women were excluded.

A 7-day estimated food diary was used to measure food intake. Food diaries were analysed using WISP ${ }^{\circledR}$ (Tinuviel Software, Warrington, UK). WISP ${ }^{\circledR}$ uses McCance \& Widdowson's The Composition of Foods, fifth edition ${ }^{22}$ and supplemental volumes ${ }^{23-31}$ along with additional data (manufacturers' data on generic foods, nutritional supplements and on new products that are commonly consumed) to determine nutrient intakes. Data manipulation and statistical analysis of the data were conducted using SPSS $^{\circledR}$ for Windows ${ }^{\text {TM }}$ Version 9.0 (SPSS Inc., Chicago, IL).

Differences between mean intakes for men and women in corresponding age groups were assessed using a nonparametric Mann-Whitney test. Differences between age groups within each sex were evaluated using a nonparametric Kruskal-Wallis test. Values of $P<0.01$ were taken as statistically significant. The percentage contribution of food groups to vitamin intakes was calculated for men and women. Owing to the large sample size, even a small difference between group means was highly statistically significant. Therefore, greater emphasis was placed on a descriptive, rather than a formal statistical analysis of these data.

\section{Results}

The mean daily intakes of vitamins from food sources and supplements by age and sex are shown in Tables 1-15. Daily intakes of vitamins expressed per $10 \mathrm{MJ}$ for men and women of all ages are shown in Table 16. In Table 17, the proportion of the population with mean daily vitamin intakes below the average requirement (AR) is reported and in Table 18 the proportion of the population with mean daily intakes below the lowest threshold intake (LTI) is reported.

\section{Retinol}

The mean daily intake was significantly higher $(P<$ $0.001)$ in men $(598 \mu \mathrm{g})$ than in women $(530 \mu \mathrm{g})$. The mean daily intakes of retinol were lowest in the 18-35 year age group for both men and women (Table 1). The primary sources of retinol in the diet for the total population were milk \& yoghurt (26.6\%), butter, spreading fats \& oils (13.0\%) and eggs \& egg dishes (10.5\%). Nutritional supplements contributed $6.7 \%$ and $10.8 \%$ to the mean daily intake of men and women, respectively.

\section{Carotene}

Table 2 shows that mean daily intakes of carotene were higher, but not significantly, in men $(2543 \mu \mathrm{g})$ of all ages compared with women $(2312 \mu \mathrm{g})$. The primary sources of carotene in the total population were vegetables \& vegetable dishes $(59.8 \%)$ and meat \& meat products (14.3\%).

\section{Total vitamin $A$}

Table 3 shows that the mean daily intakes of total vitamin A were significantly higher $(P<0.001)$ in men than in women of all ages and that mean daily intakes of total vitamin A were lowest in the 18-35 year age group for both men and women. The main contributors of total vitamin A to the diet of the total population were vegetables \& vegetable dishes $(30.7 \%)$, milk \& yoghurt 
(14.2\%) and meat \& meat products (11.5\%). Mean daily intakes of total vitamin A were less than the AR in 16.6\% of women and $20.2 \%$ of men (Table 17) and mean daily intakes of total vitamin A were less than the LTI in 3.8\% of women and $4.5 \%$ of men (Table 18 ).

\section{Vitamin D}

Mean daily intakes of vitamin $\mathrm{D}$ increased significantly $(P<0.001)$ with age for men and women (Table 4). Meat $\&$ meat products $(30.6 \%)$, fish \& fish products $(14.3 \%)$ and eggs \& egg dishes (12.3\%) were the main contributors to vitamin $\mathrm{D}$ intakes in the total population. Nutritional supplements provided $6.2 \%$ in men and $11.4 \%$ in women to the mean daily intakes of vitamin $\mathrm{D}$.

\section{Vitamin E}

Mean daily intakes of vitamin $\mathrm{E}$ for men and women were not significantly different. The mean daily intakes of vitamin $\mathrm{E}$ were lowest for men in the 51-64 year age group and for women in the 18-35 year age group (Table 5). Vegetables \& vegetable dishes (18.9\%) and potatoes \& potato products (12.4\%) were the largest contributors of vitamin $\mathrm{E}$ to the diet. Nutritional supplements contributed $5.5 \%$ to the mean daily intakes in men and $11.9 \%$ in women. Mean daily intakes of vitamin $\mathrm{E}$ were less than the LTI in $16.3 \%$ of men and $10.7 \%$ of women (Table 18 ).

\section{Thiamin}

Mean daily intakes of thiamin were higher in men than in women for all groups except the 36-50 year age group, where mean daily intake was higher in women. The main sources of thiamin in the diet in the total population were meat \& meat products (21.2\%), potatoes \& potato products $(18.5 \%)$, bread \& rolls (17.1\%) and breakfast cereals (9.9\%). Nutritional supplements contributed 3.5\% and $7.3 \%$ to the mean daily intakes in men and women, respectively. Mean daily thiamin intakes were less than the AR in $0.9 \%$ of men and $0.6 \%$ of women.

\section{Riboflavin}

Mean daily intakes of riboflavin were significantly higher $(P<0.001)$ for men than for women of all ages and decreased with age in men $(P<0.01)$. Milk \& yoghurt (24.1\%), meat \& meat products (17.5\%) and breakfast cereals $(11.3 \%)$ were the main contributors to the mean daily intakes of the total population. The AR of $1.3 \mathrm{mg}$ for men and $1.1 \mathrm{mg}$ for women is not achieved by $12.5 \%$ of men and $20.6 \%$ of women (Table 17). A much lower proportion, $0.3 \%$ of men and $1.3 \%$ of women, had mean daily riboflavin intakes below the LTI (Table 18).

\section{Niacin}

Niacin intake is estimated as both pre-formed niacin and total niacin equivalents. Mean daily intakes of pre-formed niacin and total niacin equivalents were significantly higher for men than for women for all age groups $(P<$
$0.001)$ and decreased significantly $(P<0.001)$ with age in men (Tables 8 and 9). The primary sources of pre-formed niacin and total niacin equivalents were meat $\&$ meat products $(38.5 \%$ and $36.6 \%$, respectively) and bread \& rolls ( $13.9 \%$ and $13.6 \%$, respectively). Mean daily intakes of total niacin equivalents were below the AR in $0.2 \%$ of men and $0.1 \%$ of women (Table 17) and below the LTI in $0.2 \%$ of men and $0.0 \%$ of women (Table 18 ).

\section{Vitamin $B_{6}$}

Mean daily intakes of vitamin $\mathrm{B}_{6}$ were highest in 36-50year-old women (Table 10). The major sources of vitamin $\mathrm{B}_{6}$ for the total population were potatoes \& potato products (25.1\%) and meat \& meat products (21.6\%). Nutritional supplements contributed $3.6 \%$ and $7.7 \%$ to the mean daily intake for men and women, respectively. Mean daily intakes of vitamin $B_{6}$ were below the AR in $1.7 \%$ of men and $2.4 \%$ of women.

\section{Vitamin $B_{12}$}

The mean daily intakes of vitamin $\mathrm{B}_{12}$ (Table 11) were significantly higher $(P<0.001)$ in men $\left(5.4 \mu \mathrm{g} \mathrm{day}^{-1}\right)$ than in women $\left(4.1 \mu \mathrm{g} \mathrm{day}^{-1}\right)$ for all age groups. Mean daily intakes of vitamin $B_{12}$ increased with age in men and the lowest intakes in women were observed in the 18-35 year age group. The main sources of vitamin $B_{12}$ in the diet were meat $\&$ meat products $(38.2 \%)$, milk \& yoghurt $(24.2 \%)$ and fish \& fish products (13.4\%). Mean daily intakes of vitamin $\mathrm{B}_{12}$ were below the $\mathrm{AR}$ in $0.5 \%$ of men and $0.8 \%$ of women (Table 17) and below the LTI in $0.3 \%$ of men and $0.4 \%$ of women (Table 18 ).

\section{Folate}

The mean daily intakes of folate (Table 12) were significantly higher $(P<0.001)$ in men than in women for all age groups. The primary contributors of folate in the diet of men were potatoes \& potato products $(20.0 \%)$, bread \& rolls (12.5\%), alcoholic beverages (11.5\%), vegetables \& vegetable dishes (10.9\%) and breakfast cereals $(10.1 \%)$. In women the main contributors were potatoes \& potato products $(15.3 \%)$, vegetables \& vegetable dishes $(13.6 \%)$, bread \& rolls $(12.2 \%)$ and breakfast cereals (12.0\%). Nutritional supplements contributed $2.7 \%$ and $6.4 \%$ to the mean daily folate intake for men and women, respectively. The proportion of the population with mean daily folate intakes less than the AR was $2.4 \%$ for men of all ages and $8.8 \%$ for women of all ages (Table 17), while mean daily folate intakes were below the LTI for $0.2 \%$ of men and $1.0 \%$ of women.

\section{Biotin and pantotbenate}

Mean daily intakes of biotin and pantothenate were significantly higher in men than in women for all age groups (Tables 13 and 14). The primary sources of pantothenate were meat $\&$ meat products $(26.4 \%)$, milk \& yoghurt $(15.6 \%)$, potatoes \& potato products $(12.7 \%)$ and 
Table 1 Mean daily intakes of retinol $(\mu \mathrm{g})$ from all sources by age and sex

\begin{tabular}{|c|c|c|c|c|c|c|c|c|}
\hline & \multicolumn{4}{|c|}{ Men } & \multicolumn{4}{|c|}{ Women } \\
\hline & $\begin{array}{c}18-35 \text { years } \\
n=253\end{array}$ & $\begin{array}{c}36-50 \text { years } \\
n=236\end{array}$ & $\begin{array}{c}51-64 \text { years } \\
n=173\end{array}$ & $\begin{array}{l}\text { All ages } \\
n=662\end{array}$ & $\begin{array}{c}18-35 \text { years } \\
n=269\end{array}$ & $\begin{array}{c}36-50 \text { years } \\
n=286\end{array}$ & $\begin{array}{c}51-64 \text { years } \\
n=162\end{array}$ & $\begin{array}{l}\text { All ages } \\
n=717\end{array}$ \\
\hline $\begin{array}{l}\text { Mean } \\
\text { SD } \\
\text { Median } \\
\text { Percentiles }\end{array}$ & $\begin{array}{l}487 \\
474 \\
361\end{array}$ & $\begin{array}{l}658 \\
903 \\
429\end{array}$ & $\begin{array}{l}678 \\
974 \\
405\end{array}$ & $\begin{array}{l}598^{a} \\
794 \\
397\end{array}$ & $\begin{array}{l}475 \\
528 \\
297\end{array}$ & $\begin{array}{l}560 \\
803 \\
331\end{array}$ & $\begin{array}{l}569 \\
696 \\
299\end{array}$ & $\begin{array}{l}530 \\
687 \\
315\end{array}$ \\
\hline $\begin{array}{l}\text { 5th } \\
\text { 95th }\end{array}$ & $\begin{array}{r}112 \\
1309\end{array}$ & $\begin{array}{r}135 \\
1595\end{array}$ & $\begin{array}{r}99 \\
2784\end{array}$ & $\begin{array}{r}118 \\
1521\end{array}$ & $\begin{array}{r}94 \\
1300\end{array}$ & $\begin{array}{r}92 \\
1961\end{array}$ & $\begin{array}{r}82 \\
2354\end{array}$ & $\begin{array}{r}90 \\
1740\end{array}$ \\
\hline
\end{tabular}

${ }^{a}$ Significantly different from women, $P<0.001$.

Table 2 Mean daily intakes of carotene $(\mu \mathrm{g})$ from all sources by age and sex

\begin{tabular}{|c|c|c|c|c|c|c|c|c|}
\hline & \multicolumn{4}{|c|}{ Men } & \multicolumn{4}{|c|}{ Women } \\
\hline & $\begin{array}{c}18-35 \text { years } \\
n=253\end{array}$ & $\begin{array}{c}36-50 \text { years } \\
n=236\end{array}$ & $\begin{array}{c}51-64 \text { years } \\
n=173\end{array}$ & $\begin{array}{l}\text { All ages } \\
n=662\end{array}$ & $\begin{array}{c}18-35 \text { years } \\
n=269\end{array}$ & $\begin{array}{c}36-50 \text { years } \\
n=286\end{array}$ & $\begin{array}{c}51-64 \text { years } \\
n=162\end{array}$ & $\begin{array}{l}\text { All ages } \\
n=717\end{array}$ \\
\hline $\begin{array}{l}\text { Mean } \\
\text { SD } \\
\text { Median } \\
\text { Percentiles }\end{array}$ & $\begin{array}{l}2256 \\
2388 \\
1807\end{array}$ & $\begin{array}{l}2891^{a} \\
1961 \\
2580\end{array}$ & $\begin{array}{l}2489 \\
1704 \\
2167\end{array}$ & $\begin{array}{l}2543 \\
2091 \\
2087\end{array}$ & $\begin{array}{l}1971 \\
1496 \\
1609\end{array}$ & $\begin{array}{l}2432 \\
1594 \\
2017\end{array}$ & $\begin{array}{l}2668 \\
1858 \\
2130\end{array}$ & $\begin{array}{l}2312 \\
1644 \\
1913\end{array}$ \\
\hline $\begin{array}{l}\text { 5th } \\
\text { 95th }\end{array}$ & $\begin{array}{r}403 \\
4990\end{array}$ & $\begin{array}{r}449 \\
6082\end{array}$ & $\begin{array}{r}359 \\
5597\end{array}$ & $\begin{array}{r}409 \\
5807\end{array}$ & $\begin{array}{r}364 \\
4504\end{array}$ & $\begin{array}{r}573 \\
5325\end{array}$ & $\begin{array}{r}536 \\
6568\end{array}$ & $\begin{array}{r}480 \\
5410\end{array}$ \\
\hline
\end{tabular}

a Significantly different from women, $P<0.01$.

Differences in mean intakes between age groups were significant for men and women, $P<0.01$.

Table 3 Mean daily intakes of total vitamin A $(\mu \mathrm{g})$ from all sources by age and sex

\begin{tabular}{|c|c|c|c|c|c|c|c|c|}
\hline & \multicolumn{4}{|c|}{ Men } & \multicolumn{4}{|c|}{ Women } \\
\hline & $\begin{array}{c}18-35 \text { years } \\
n=253\end{array}$ & $\begin{array}{c}36-50 \text { years } \\
n=236\end{array}$ & $\begin{array}{c}51-64 \text { years } \\
n=173\end{array}$ & $\begin{array}{l}\text { All ages } \\
n=662\end{array}$ & $\begin{array}{c}18-35 \text { years } \\
n=269\end{array}$ & $\begin{array}{c}36-50 \text { years } \\
n=286\end{array}$ & $\begin{array}{c}51-64 \text { years } \\
n=162\end{array}$ & $\begin{array}{l}\text { All ages } \\
n=717\end{array}$ \\
\hline $\begin{array}{l}\text { Mean } \\
\text { SD } \\
\text { Median } \\
\text { Percentiles }\end{array}$ & $\begin{array}{l}863 \\
643 \\
719\end{array}$ & $\begin{array}{c}1140^{\mathrm{a}} \\
978 \\
966\end{array}$ & $\begin{array}{r}1093 \\
1037 \\
832\end{array}$ & $\begin{array}{c}1022^{\mathrm{a}} \\
891 \\
823\end{array}$ & $\begin{array}{l}804 \\
608 \\
647\end{array}$ & $\begin{array}{l}965 \\
831 \\
751\end{array}$ & $\begin{array}{r}1013 \\
799 \\
752\end{array}$ & $\begin{array}{l}915 \\
751 \\
720\end{array}$ \\
\hline $\begin{array}{l}5 \text { th } \\
95 \text { th }\end{array}$ & $\begin{array}{r}273 \\
1859\end{array}$ & $\begin{array}{r}352 \\
2310\end{array}$ & $\begin{array}{r}311 \\
3274\end{array}$ & $\begin{array}{r}310 \\
2293\end{array}$ & $\begin{array}{r}228 \\
1904\end{array}$ & $\begin{array}{r}274 \\
2284\end{array}$ & $\begin{array}{r}292 \\
2858\end{array}$ & $\begin{array}{r}264 \\
2429\end{array}$ \\
\hline
\end{tabular}

${ }^{a}$ Significantly different from women, $P<0.001$.

Differences in mean intake between age groups were significant for men, $P<0.01$, and women, $P<0.001$.

Table 4 Mean daily intakes of vitamin $D(\mu \mathrm{g})$ from all sources by age and sex

\begin{tabular}{|c|c|c|c|c|c|c|c|c|}
\hline & \multicolumn{4}{|c|}{ Men } & \multicolumn{4}{|c|}{ Women } \\
\hline & $\begin{array}{c}18-35 \text { years } \\
n=253\end{array}$ & $\begin{array}{c}36-50 \text { years } \\
n=236\end{array}$ & $\begin{array}{c}51-64 \text { years } \\
n=173\end{array}$ & $\begin{array}{l}\text { All ages } \\
n=662\end{array}$ & $\begin{array}{c}18-35 \text { years } \\
n=269\end{array}$ & $\begin{array}{c}36-50 \text { years } \\
n=286\end{array}$ & $\begin{array}{c}51-64 \text { years } \\
n=162\end{array}$ & $\begin{array}{l}\text { All ages } \\
n=717\end{array}$ \\
\hline $\begin{array}{l}\text { Mean } \\
\text { SD } \\
\text { Median } \\
\text { Percentiles }\end{array}$ & $\begin{array}{l}3.0^{\mathrm{a}} \\
2.5 \\
2.1\end{array}$ & $\begin{array}{l}3.9^{a} \\
3.2 \\
2.8\end{array}$ & $\begin{array}{l}4.4 \\
4.5 \\
3.0\end{array}$ & $\begin{array}{l}3.7 \\
3.4 \\
2.5\end{array}$ & $\begin{array}{l}2.8 \\
3.1 \\
1.8\end{array}$ & $\begin{array}{l}3.4 \\
3.3 \\
2.1\end{array}$ & $\begin{array}{r}5.8 \\
17.2 \\
2.7\end{array}$ & $\begin{array}{l}3.7 \\
8.7 \\
2.1\end{array}$ \\
\hline $\begin{array}{l}\text { 5th } \\
\text { 95th }\end{array}$ & $\begin{array}{l}0.9 \\
8.1\end{array}$ & $\begin{array}{r}1.1 \\
11.4\end{array}$ & $\begin{array}{r}1.1 \\
13.9\end{array}$ & $\begin{array}{r}1.0 \\
10.5\end{array}$ & $\begin{array}{l}0.6 \\
7.8\end{array}$ & $\begin{array}{r}0.7 \\
11.3\end{array}$ & $\begin{array}{r}0.6 \\
17.0\end{array}$ & $\begin{array}{r}0.6 \\
11.2\end{array}$ \\
\hline
\end{tabular}

a Significantly different from women, $P<0.001$.

Differences between age groups were significant for men and women, $P<0.001$.

bread \& rolls (9.2\%). Nutritional supplements contributed $3.2 \%$ in men and $6.1 \%$ in women. The primary sources of biotin were non-alcoholic beverages (19.6\%), biscuits, cakes \& pastries $(14.0 \%)$ and meat \& meat products (13.1\%). Nutritional supplements contributed $1.7 \%$ in men and $3.6 \%$ in women.
Vitamin C

Mean daily intakes of vitamin $\mathrm{C}$ were not significantly different in men $(116 \mathrm{mg})$ and women $(108 \mathrm{mg})$. The primary sources of vitamin $\mathrm{C}$ for the total population were potatoes \& potato products (25.9\%), fruit, juice, nuts \& seeds, herbs \& spices (25.2\%) and vegetables $\&$ vegetable 
Table 5 Mean daily intakes of vitamin $E(\mathrm{mg})$ from all sources by age and sex

\begin{tabular}{|c|c|c|c|c|c|c|c|c|}
\hline & \multicolumn{4}{|c|}{ Men } & \multicolumn{4}{|c|}{ Women } \\
\hline & $\begin{array}{c}18-35 \text { years } \\
n=253\end{array}$ & $\begin{array}{c}36-50 \text { years } \\
n=236\end{array}$ & $\begin{array}{c}51-64 \text { years } \\
n=173\end{array}$ & $\begin{array}{l}\text { All ages } \\
n=662\end{array}$ & $\begin{array}{c}18-35 \text { years } \\
n=269\end{array}$ & $\begin{array}{c}36-50 \text { years } \\
n=286\end{array}$ & $\begin{array}{c}51-64 \text { years } \\
n=162\end{array}$ & $\begin{array}{l}\text { All ages } \\
n=717\end{array}$ \\
\hline $\begin{array}{l}\text { Mean } \\
\text { SD } \\
\text { Median } \\
\text { Percentiles }\end{array}$ & $\begin{array}{r}12.2 \\
47.6 \\
6.7\end{array}$ & $\begin{array}{r}12.3 \\
34.0 \\
6.4\end{array}$ & $\begin{array}{r}8.1 \\
20.5 \\
5.8\end{array}$ & $\begin{array}{r}11.2 \\
37.2 \\
6.3\end{array}$ & $\begin{array}{r}8.9 \\
17.7 \\
6.1\end{array}$ & $\begin{array}{r}12.2 \\
29.7 \\
6.3\end{array}$ & $\begin{array}{r}12.3 \\
35.2 \\
5.4\end{array}$ & $\begin{array}{r}11.0 \\
27.4 \\
6.0\end{array}$ \\
\hline $\begin{array}{l}\text { 5th } \\
\text { 95th }\end{array}$ & $\begin{array}{r}2.6 \\
19.6\end{array}$ & $\begin{array}{r}2.5 \\
20.2\end{array}$ & $\begin{array}{r}2.1 \\
15.0\end{array}$ & $\begin{array}{r}2.5 \\
17.9\end{array}$ & $\begin{array}{r}2.5 \\
17.8\end{array}$ & $\begin{array}{r}2.4 \\
33.7\end{array}$ & $\begin{array}{r}1.7 \\
26.1\end{array}$ & $\begin{array}{r}2.1 \\
23.6\end{array}$ \\
\hline
\end{tabular}

Differences between age groups were significant for men only, $P<0.001$.

Table 6 Mean daily intakes of thiamin $(\mathrm{mg})$ from all sources by age and sex

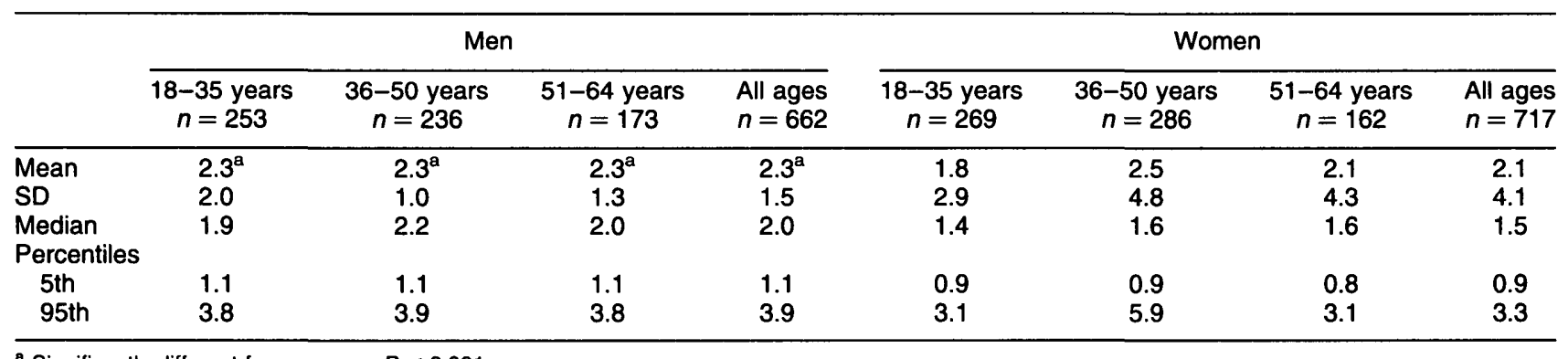

Significantly different from women, $P<0.001$.

Differences between age groups were significant for men only, $P<0.01$.

Table 7 Mean daily intakes of riboflavin $(\mathrm{mg})$ from all sources by age and sex

\begin{tabular}{|c|c|c|c|c|c|c|c|c|}
\hline & \multicolumn{4}{|c|}{ Men } & \multicolumn{4}{|c|}{ Women } \\
\hline & $\begin{array}{c}18-35 \text { years } \\
n=253\end{array}$ & $\begin{array}{c}36-50 \text { years } \\
n=236\end{array}$ & $\begin{array}{c}51-64 \text { years } \\
n=173\end{array}$ & $\begin{array}{l}\text { All ages } \\
n=662\end{array}$ & $\begin{array}{c}18-35 \text { years } \\
n=269\end{array}$ & $\begin{array}{c}36-50 \text { years } \\
n=286\end{array}$ & $\begin{array}{c}51-64 \text { years } \\
n=162\end{array}$ & $\begin{array}{l}\text { All ages } \\
n=717\end{array}$ \\
\hline Mean & $2.3^{\mathrm{a}}$ & $2.2^{a}$ & $2.0^{2}$ & $2.2^{\mathrm{a}}$ & 1.8 & 2.4 & 1.8 & 2.0 \\
\hline SD & 2.1 & 1.0 & 0.8 & 1.5 & 2.6 & 4.7 & 0.9 & 3.4 \\
\hline Median & 2.0 & 2.1 & 1.8 & 2.0 & 1.4 & 1.6 & 1.5 & 1.5 \\
\hline \multicolumn{9}{|l|}{ Percentiles } \\
\hline 5th & 1.1 & 1.0 & 1.1 & 1.0 & 0.7 & 0.8 & 0.8 & 0.8 \\
\hline 95th & 3.9 & 4.0 & 3.5 & 3.9 & 3.6 & 4.3 & 3.4 & 3.7 \\
\hline
\end{tabular}

a Significantly different from women, $P<0.001$.

Differences between age groups were significant for men only, $P<0.01$.

Table 8 Mean daily intakes of pre-formed niacin $(\mathrm{mg})$ from all sources by age and sex

\begin{tabular}{|c|c|c|c|c|c|c|c|c|}
\hline & \multicolumn{4}{|c|}{ Men } & \multicolumn{4}{|c|}{ Women } \\
\hline & $\begin{array}{c}18-35 \text { years } \\
n=253\end{array}$ & $\begin{array}{c}36-50 \text { years } \\
n=236\end{array}$ & $\begin{array}{c}51-64 \text { years } \\
n=173\end{array}$ & $\begin{array}{l}\text { All ages } \\
n=662\end{array}$ & $\begin{array}{c}18-35 \text { years } \\
n=269\end{array}$ & $\begin{array}{c}36-50 \text { years } \\
n=286\end{array}$ & $\begin{array}{c}51-64 \text { years } \\
n=162\end{array}$ & $\begin{array}{l}\text { All ages } \\
n=717\end{array}$ \\
\hline $\begin{array}{l}\text { Mean } \\
\text { SD } \\
\text { Median } \\
\text { Percentiles }\end{array}$ & $\begin{array}{c}29.6^{\mathrm{a}} \\
9.9 \\
27.9\end{array}$ & $\begin{array}{c}28.4^{\mathrm{a}} \\
9.9 \\
27.0\end{array}$ & $\begin{array}{c}25.8^{\mathrm{a}} \\
9.4 \\
24.8\end{array}$ & $\begin{array}{c}28.2^{\mathrm{a}} \\
9.9 \\
26.9\end{array}$ & $\begin{array}{r}20.1 \\
8.6 \\
18.4\end{array}$ & $\begin{array}{l}21.9 \\
11.6 \\
19.5\end{array}$ & $\begin{array}{r}19.4 \\
8.4 \\
17.6\end{array}$ & $\begin{array}{r}20.7 \\
9.9 \\
18.9\end{array}$ \\
\hline $\begin{array}{l}\text { 5th } \\
\text { 95th }\end{array}$ & $\begin{array}{l}15.5 \\
46.9\end{array}$ & $\begin{array}{l}15.4 \\
48.1\end{array}$ & $\begin{array}{l}13.6 \\
43.5\end{array}$ & $\begin{array}{l}15.0 \\
46.6\end{array}$ & $\begin{array}{l}10.1 \\
35.5\end{array}$ & $\begin{array}{l}11.6 \\
40.9\end{array}$ & $\begin{array}{r}8.7 \\
36.7\end{array}$ & $\begin{array}{l}10.5 \\
37.6\end{array}$ \\
\hline
\end{tabular}

a Significantly different from women, $P<0.001$.

Differences between age groups were significant for men only, $P<0.001$.

dishes (22.1\%). The contribution from supplements was $5.8 \%$ for men and $8.3 \%$ for women. Table 17 shows that $7.6 \%$ of men and $8.6 \%$ of women of all ages did not achieve the AR of $30 \mathrm{mg}^{\text {day }}{ }^{-1}$ of vitamin $\mathrm{C}$ while $0.3 \%$ of men and $0.4 \%$ of women had mean daily vitamin $C$ intakes below the LTI of $12 \mathrm{mg}^{\text {day }}{ }^{-1}$ (Table 18).

\section{Nutrient density}

Table 16 shows the nutrient density of the diet in men and women by age group. The mean nutrient density of dietary intake was generally higher for most vitamins for women and significantly so for some, e.g. total vitamin A and vitamin C. Nutrient density generally increased with 
Table 9 Mean daily intakes of total niacin equivalents $(\mathrm{mg})$ from all sources by age and sex

\begin{tabular}{|c|c|c|c|c|c|c|c|c|}
\hline & \multicolumn{4}{|c|}{ Men } & \multicolumn{4}{|c|}{ Women } \\
\hline & $\begin{array}{c}18-35 \text { years } \\
n=253\end{array}$ & $\begin{array}{c}36-50 \text { years } \\
n=236\end{array}$ & $\begin{array}{c}51-64 \text { years } \\
n=173\end{array}$ & $\begin{array}{l}\text { All ages } \\
n=662\end{array}$ & $\begin{array}{c}18-35 \text { years } \\
n=269\end{array}$ & $\begin{array}{c}36-50 \text { years } \\
n=286\end{array}$ & $\begin{array}{c}51-64 \text { years } \\
n=162\end{array}$ & $\begin{array}{l}\text { All ages } \\
n=717\end{array}$ \\
\hline $\begin{array}{l}\text { Mean } \\
\text { SD } \\
\text { Median } \\
\text { Percentiles }\end{array}$ & $\begin{array}{l}50.8^{a} \\
14.5 \\
50.1\end{array}$ & $\begin{array}{l}49.5^{a} \\
14.7 \\
47.4\end{array}$ & $\begin{array}{l}45.6^{a} \\
12.6 \\
43.8\end{array}$ & $\begin{array}{l}49.0^{a} \\
14.2 \\
47.4\end{array}$ & $\begin{array}{l}33.7 \\
11.0 \\
32.6\end{array}$ & $\begin{array}{l}36.6 \\
13.7 \\
34.4\end{array}$ & $\begin{array}{l}33.7 \\
10.5 \\
32.9\end{array}$ & $\begin{array}{l}34.9 \\
12.1 \\
33.5\end{array}$ \\
\hline $\begin{array}{l}\text { 5th } \\
\text { 95th }\end{array}$ & $\begin{array}{l}28.6 \\
75.6\end{array}$ & $\begin{array}{l}29.4 \\
76.7\end{array}$ & $\begin{array}{l}29.4 \\
73.9\end{array}$ & $\begin{array}{l}29.2 \\
75.4\end{array}$ & $\begin{array}{l}19.5 \\
54.0\end{array}$ & $\begin{array}{l}21.6 \\
58.7\end{array}$ & $\begin{array}{l}17.3 \\
53.2\end{array}$ & $\begin{array}{l}20.1 \\
54.3\end{array}$ \\
\hline
\end{tabular}

a Significantly different from women, $P<0.001$.

Differences between age groups were significant for men only, $P<0.001$.

Table 10 Mean daily intakes of vitamin $B_{6}(\mathrm{mg})$ from all sources by age and sex

\begin{tabular}{|c|c|c|c|c|c|c|c|c|}
\hline & \multicolumn{4}{|c|}{ Men } & \multicolumn{4}{|c|}{ Women } \\
\hline & $\begin{array}{c}18-35 \text { years } \\
n=253\end{array}$ & $\begin{array}{c}36-50 \text { years } \\
n=236\end{array}$ & $\begin{array}{c}51-64 \text { years } \\
n=173\end{array}$ & $\begin{array}{l}\text { All ages } \\
n=662\end{array}$ & $\begin{array}{c}18-35 \text { years } \\
n=269\end{array}$ & $\begin{array}{c}36-50 \text { years } \\
n=286\end{array}$ & $\begin{array}{c}51-64 \text { years } \\
n=162\end{array}$ & $\begin{array}{l}\text { All ages } \\
n=717\end{array}$ \\
\hline $\begin{array}{l}\text { Mean } \\
\text { SD } \\
\text { Median } \\
\text { Percentiles }\end{array}$ & $\begin{array}{l}3.6^{a} \\
2.5 \\
3.1\end{array}$ & $\begin{array}{l}3.4^{a} \\
1.6 \\
3.2\end{array}$ & $\begin{array}{l}3.2^{\mathrm{a}} \\
1.3 \\
2.9\end{array}$ & $\begin{array}{l}3.5^{\mathrm{a}} \\
1.9 \\
3.1\end{array}$ & $\begin{array}{l}2.9 \\
5.2 \\
2.1\end{array}$ & $\begin{array}{l}3.8 \\
7.4 \\
2.1\end{array}$ & $\begin{array}{l}3.1 \\
6.2 \\
2.1\end{array}$ & $\begin{array}{l}3.3 \\
6.4 \\
2.1\end{array}$ \\
\hline $\begin{array}{l}\text { 5th } \\
\text { 95th }\end{array}$ & $\begin{array}{l}1.8 \\
6.9\end{array}$ & $\begin{array}{l}1.6 \\
6.4\end{array}$ & $\begin{array}{l}1.8 \\
5.9\end{array}$ & $\begin{array}{l}1.8 \\
6.3\end{array}$ & $\begin{array}{l}1.2 \\
5.2\end{array}$ & $\begin{array}{r}1.2 \\
12.3\end{array}$ & $\begin{array}{l}1.0 \\
4.5\end{array}$ & $\begin{array}{l}1.2 \\
5.5\end{array}$ \\
\hline
\end{tabular}

${ }^{a}$ Significantly different from women, $P<0.001$.

Table 11 Mean daily intakes of vitamin $B_{12}(\mu \mathrm{g})$ from all sources by age and sex

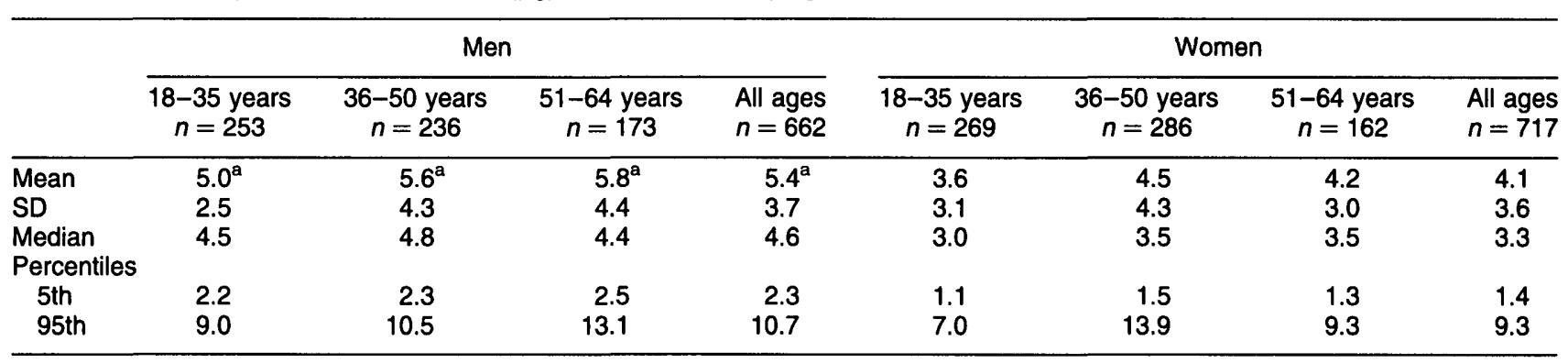

a Significantly different from women, $P<0.001$.

Differences between age groups were significant for women only, $P<0.01$.

Table 12 Mean daily intakes of folate $(\mu \mathrm{g})$ from all sources by age and sex

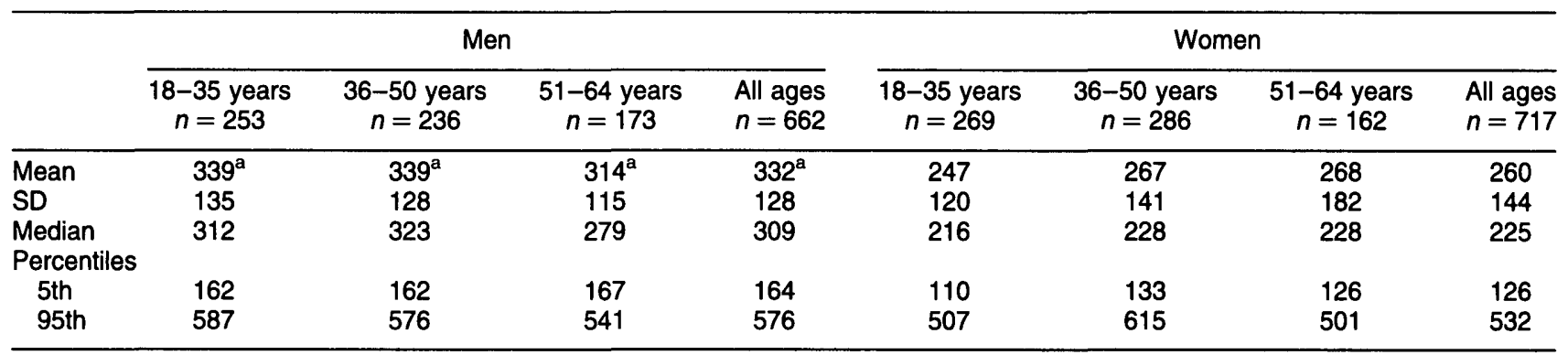

${ }^{a}$ Significantly different from women, $P<0.001$.

age for men and women except for vitamin $C$, which decreased in men (Table 16).

\section{Discussion}

The problem of assessing the adequacy of nutrient intakes at the population level has been a long-standing one ${ }^{32}$.
The Recommended Dietary Amount (RDA) for a nutrient has been used as a cut-off value to assess the prevalence of nutrient inadequacy; however, this approach leads to overestimation of nutrient inadequacy. While at an individual level it is reasonable to compare an individual's intake to the RDA, at the population level it is difficult to justify doing $\mathrm{so}^{32}$. 
Table 13 Mean daily intakes of biotin $(\mu \mathrm{g})$ from all sources by age and sex

\begin{tabular}{|c|c|c|c|c|c|c|c|c|}
\hline & \multicolumn{4}{|c|}{ Men } & \multicolumn{4}{|c|}{ Women } \\
\hline & $\begin{array}{c}18-35 \text { years } \\
n=253\end{array}$ & $\begin{array}{c}36-50 \text { years } \\
n=236\end{array}$ & $\begin{array}{c}51-64 \text { years } \\
n=173\end{array}$ & $\begin{array}{l}\text { All ages } \\
n=662\end{array}$ & $\begin{array}{c}18-35 \text { years } \\
n=269\end{array}$ & $\begin{array}{c}36-50 \text { years } \\
n=286\end{array}$ & $\begin{array}{c}51-64 \text { years } \\
n=162\end{array}$ & $\begin{array}{l}\text { All ages } \\
n=717\end{array}$ \\
\hline $\begin{array}{l}\text { Mean } \\
\text { SD } \\
\text { Median } \\
\text { Percentiles }\end{array}$ & $\begin{array}{l}40.6^{a} \\
21.4 \\
37.6\end{array}$ & $\begin{array}{l}45.6^{a} \\
26.3 \\
41.9\end{array}$ & $\begin{array}{l}42.4^{a} \\
22.4 \\
38.3\end{array}$ & $\begin{array}{l}42.8^{a} \\
23.6 \\
38.9\end{array}$ & $\begin{array}{l}32.5 \\
31.1 \\
26.2\end{array}$ & $\begin{array}{l}35.7 \\
20.4 \\
31.0\end{array}$ & $\begin{array}{l}34.0 \\
20.7 \\
31.0\end{array}$ & $\begin{array}{l}34.1 \\
25.0 \\
29.3\end{array}$ \\
\hline $\begin{array}{l}\text { 5th } \\
95 \text { th }\end{array}$ & $\begin{array}{l}18.6 \\
67.8\end{array}$ & $\begin{array}{l}20.3 \\
81.8\end{array}$ & $\begin{array}{l}22.0 \\
71.5\end{array}$ & $\begin{array}{l}20.2 \\
73.2\end{array}$ & $\begin{array}{l}12.6 \\
64.8\end{array}$ & $\begin{array}{l}17.9 \\
66.9\end{array}$ & $\begin{array}{l}16.4 \\
55.8\end{array}$ & $\begin{array}{l}15.5 \\
64.0\end{array}$ \\
\hline
\end{tabular}

Significantly different from women, $P<0.001$.

Differences between age groups were significant for women only, $P<0.001$.

Table 14 Mean daily intakes of pantothenate $(\mathrm{mg})$ from all sources by age and sex

\begin{tabular}{|c|c|c|c|c|c|c|c|c|}
\hline & \multicolumn{4}{|c|}{ Men } & \multicolumn{4}{|c|}{ Women } \\
\hline & $\begin{array}{c}18-35 \text { years } \\
n=253\end{array}$ & $\begin{array}{c}36-50 \text { years } \\
n=236\end{array}$ & $\begin{array}{c}51-64 \text { years } \\
n=173\end{array}$ & $\begin{array}{l}\text { All ages } \\
n=662\end{array}$ & $\begin{array}{c}18-35 \text { years } \\
n=269\end{array}$ & $\begin{array}{c}36-50 \text { years } \\
n=286\end{array}$ & $\begin{array}{c}51-64 \text { years } \\
n=162\end{array}$ & $\begin{array}{l}\text { All ages } \\
n=717\end{array}$ \\
\hline $\begin{array}{l}\text { Mean } \\
\text { SD } \\
\text { Median } \\
\text { Percentiles }\end{array}$ & $\begin{array}{l}6.4^{\mathrm{a}} \\
2.9 \\
5.7\end{array}$ & $\begin{array}{l}6.8^{\mathrm{a}} \\
2.9 \\
6.3\end{array}$ & $\begin{array}{l}6.3^{\mathrm{a}} \\
2.1 \\
5.9\end{array}$ & $\begin{array}{l}6.5^{a} \\
2.7 \\
6.0\end{array}$ & $\begin{array}{l}4.7 \\
3.6 \\
3.9\end{array}$ & $\begin{array}{l}5.9 \\
6.4 \\
4.5\end{array}$ & $\begin{array}{l}5.1 \\
2.5 \\
4.5\end{array}$ & $\begin{array}{l}5.3 \\
4.8 \\
4.3\end{array}$ \\
\hline $\begin{array}{l}\text { 5th } \\
95 \text { th }\end{array}$ & $\begin{array}{r}3.4 \\
11.1\end{array}$ & $\begin{array}{r}3.2 \\
10.9\end{array}$ & $\begin{array}{r}3.8 \\
10.6\end{array}$ & $\begin{array}{r}3.4 \\
11.0\end{array}$ & $\begin{array}{l}2.4 \\
9.2\end{array}$ & $\begin{array}{r}2.6 \\
13.5\end{array}$ & $\begin{array}{r}2.7 \\
10.7\end{array}$ & $\begin{array}{r}2.5 \\
10.5\end{array}$ \\
\hline
\end{tabular}

a Significantly different from women, $P<0.001$.

Differences between age groups were significant for women only, $P<0.001$.

Table 15 Mean daily intakes of vitamin $C(\mathrm{mg})$ from all sources by age and sex

\begin{tabular}{|c|c|c|c|c|c|c|c|c|}
\hline & \multicolumn{4}{|c|}{ Men } & \multicolumn{4}{|c|}{ Women } \\
\hline & $\begin{array}{c}18-35 \text { years } \\
n=253\end{array}$ & $\begin{array}{c}36-50 \text { years } \\
n=236\end{array}$ & $\begin{array}{c}51-64 \text { years } \\
n=173\end{array}$ & $\begin{array}{l}\text { All ages } \\
n=662\end{array}$ & $\begin{array}{c}18-35 \text { years } \\
n=269\end{array}$ & $\begin{array}{c}36-50 \text { years } \\
n=286\end{array}$ & $\begin{array}{c}51-64 \text { years } \\
n=162\end{array}$ & $\begin{array}{l}\text { All ages } \\
n=717\end{array}$ \\
\hline $\begin{array}{l}\text { Mean } \\
\text { SD } \\
\text { Median } \\
\text { Percentiles }\end{array}$ & $\begin{array}{r}126 \\
298 \\
72\end{array}$ & $\begin{array}{r}119 \\
187 \\
77\end{array}$ & $\begin{array}{r}99 \\
111 \\
75\end{array}$ & $\begin{array}{r}116 \\
223 \\
74\end{array}$ & $\begin{array}{r}99 \\
191 \\
64\end{array}$ & $\begin{array}{r}113 \\
187 \\
69\end{array}$ & $\begin{array}{r}115 \\
164 \\
75\end{array}$ & $\begin{array}{r}108 \\
183 \\
69\end{array}$ \\
\hline $\begin{array}{l}\text { 5th } \\
\text { 95th }\end{array}$ & $\begin{array}{r}23 \\
368\end{array}$ & $\begin{array}{r}28 \\
270\end{array}$ & $\begin{array}{r}29 \\
195\end{array}$ & $\begin{array}{r}26 \\
266\end{array}$ & $\begin{array}{r}22 \\
196\end{array}$ & $\begin{array}{r}26 \\
315\end{array}$ & $\begin{array}{r}24 \\
464\end{array}$ & $\begin{array}{r}24 \\
289\end{array}$ \\
\hline
\end{tabular}

In this paper, the proportion of the population with inadequate intakes of vitamins was estimated from the percentage of the population with mean daily intakes below the average requirement (AR). The average requirement (AR) is the daily intake value that is estimated to meet the requirement, as defined by a specified indicator of adequacy, in $50 \%$ of a life-stage or gender group $^{8}$. The percentage of the population with mean daily intakes below the $A R$ is an estimate of the percentage of the population with inadequate intake ${ }^{8}$. This estimate of inadequate intakes is most accurate if mean daily intakes and the mean requirements are independent, if the requirements are symmetrically but not necessarily normally distributed, and if the standard deviation (SD) of the mean intakes is at least twice as large as the SD of the average requirement. It is also important that habitual intakes are measured. Misreporting of intake data can also affect the estimate of inadequate intakes ${ }^{8}$. A detailed account of this approach to assess the prevalence of inadequacy in a population has been described elsewhere ${ }^{32}$.

In relation to the data presented in this paper, the SD of the mean intakes is much larger than the commonly assumed SD of requirement of $15 \%$ of the mean ${ }^{3}$. It is generally assumed that the intakes and requirements of nutrients are independent (but this is not true for energy) and that the distribution of the average requirement of vitamins is normal. Bingham et al ${ }^{33}$ reported that there were no significant differences between mean daily nutrient intake estimated using an open-ended 7-day food diary and a 16-day weighed record, which was validated by 24 -hour urinary excretion of nitrogen. A 7 day food diary used in this survey was thus considered a useful means of measuring habitual intake with respect to vitamins $^{33}$. However, for certain vitamins, a significant contribution to mean daily intake is made by rich dietary sources that are consumed on an irregular basis. For example, the habitual intake for an individual of retinol 
Table 16 Mean daily intakes of vitamins per $10 \mathrm{MJ}$ for men and women from all sources

\begin{tabular}{|c|c|c|c|c|c|c|c|c|c|c|c|c|}
\hline \multirow[b]{2}{*}{ Vitamin (units per $10 \mathrm{MJ}$ ) } & \multicolumn{3}{|c|}{$18-35$ years } & \multicolumn{3}{|c|}{$36-50$ years } & \multicolumn{3}{|c|}{$51-64$ years } & \multicolumn{3}{|c|}{ All ages (18-64 years) } \\
\hline & Mean & Median & SD & Mean & Median & SD & Mean & Median & SD & Mean & Median & SD \\
\hline MEN & \multicolumn{3}{|c|}{$n=253$} & \multicolumn{3}{|c|}{$n=236$} & \multicolumn{3}{|c|}{$n=173$} & \multicolumn{3}{|c|}{$n=662$} \\
\hline Retinol $(\mu \mathrm{g})$ & $423^{a}$ & 316 & 425 & 600 & 374 & 793 & 690 & 394 & 1036 & $556^{a}$ & 363 & 764 \\
\hline Carotene $(\mu \mathrm{g})$ & $1974^{\mathrm{a}}$ & 1599 & 1863 & $2687^{b}$ & 2330 & 1837 & $2587^{a}$ & 2190 & 2088 & $2389^{\mathrm{a}}$ & 1943 & 1941 \\
\hline Total vitamin A $(\mu \mathrm{g})$ & $752^{a}$ & 630 & 534 & $1048^{b}$ & 901 & 853 & $1121^{a}$ & 840 & 1133 & $954^{a}$ & 766 & 853 \\
\hline Vitamin D $(\mu \mathrm{g})$ & $2.6^{\mathrm{b}}$ & 1.9 & 2.2 & 3.6 & 2.5 & 3.0 & $4.5^{b}$ & 2.8 & 4.7 & $3.5^{\mathrm{a}}$ & 2.3 & 3.3 \\
\hline Vitamin E (mg) & $11.2^{\mathrm{a}}$ & 6.1 & 49.9 & $11.5^{\mathrm{a}}$ & 6.1 & 31.8 & $8.9^{\mathrm{a}}$ & 6.1 & 30.7 & $10.7^{\mathrm{a}}$ & 6.1 & 39.4 \\
\hline Thiamin $(\mathrm{mg})$ & $2.0^{\mathrm{a}}$ & 1.7 & 1.5 & 2.2 & 2.0 & 1.0 & 2.4 & 2.1 & 2.1 & $2.1^{\mathrm{a}}$ & 1.9 & 1.5 \\
\hline Riboflavin (mg) & 2.0 & 1.8 & 1.5 & $2.1^{\mathrm{b}}$ & 1.9 & 0.9 & $2.0^{\mathrm{a}}$ & 1.8 & 0.8 & $2.0^{a}$ & 1.8 & 1.1 \\
\hline Pre-formed niacin (mg) & 25.9 & 24.9 & 7.3 & 26.5 & 25.0 & 9.2 & 26.4 & 24.4 & 10.2 & 26.3 & 24.9 & 8.8 \\
\hline Total niacin equivalents (mg) & 44.5 & 43.8 & 9.0 & 46.0 & 44.4 & 11.1 & 46.3 & 44.5 & 12.2 & 45.5 & 44.1 & 10.7 \\
\hline Vitamin $B_{6}(m g)$ & 3.1 & 2.8 & 1.8 & 3.2 & 2.8 & 2.0 & 3.2 & 2.9 & 1.3 & 3.2 & 2.9 & 1.8 \\
\hline Vit & 4.4 & 4.0 & 2.1 & 5.2 & 4.4 & 3.8 & 6.0 & 4.6 & 4.8 & 5.1 & 4.3 & 3.6 \\
\hline Folate ( & 296 & 276 & 101 & 316 & 294 & 122 & $316^{b}$ & 294 & 103 & $308^{a}$ & 289 & 110 \\
\hline Biotin ( & 35.5 & 33.3 & 17.8 & $42.0^{b}$ & 38.3 & 23.2 & $42.0^{b}$ & 39.2 & 17.9 & $39.5^{\mathrm{a}}$ & 36.5 & 20.1 \\
\hline Pantothenate $(\mathrm{mg})$ & 5.6 & 5.1 & 2.1 & 6.3 & 5.8 & 2.8 & 6.3 & 6.1 & 1.9 & 6.0 & 5.6 & 2.4 \\
\hline Vitamin C (mg) & $122^{\mathrm{a}}$ & 62 & 384 & $115^{\mathrm{a}}$ & 72 & 191 & $103^{a}$ & 76 & 142 & 114 & 68 & 273 \\
\hline WOMEN & \multicolumn{3}{|c|}{$n=269$} & \multicolumn{3}{|c|}{$n=286$} & \multicolumn{3}{|c|}{$n=162$} & \multicolumn{3}{|c|}{$n=717$} \\
\hline $\mathbf{R e}$ & 618 & 384 & 693 & 729 & 399 & 1074 & 783 & 421 & 988 & 699 & 396 & 929 \\
\hline Caro & 2651 & 2155 & 2072 & 3260 & 2593 & 2316 & 3895 & 3189 & 2824 & 3175 & 2507 & 2400 \\
\hline Total vitamin A $(\mu \mathrm{g})$ & 1060 & 857 & 790 & 1272 & 954 & 1117 & 1432 & 1029 & 1154 & 1229 & 941 & 1025 \\
\hline in $D(\mu g)$ & 3.7 & 2.4 & 4.1 & 4.5 & 2.6 & 4.7 & 7.3 & 4.4 & 15.2 & 4.9 & 2.8 & 8.3 \\
\hline$E(\mathrm{mg})$ & 11.3 & 8.1 & 17.4 & 15.0 & 8.0 & 31.7 & 16.5 & 7.8 & 46.1 & 13.9 & 8.0 & 31.6 \\
\hline Thiamin $(\mathrm{mg})$ & 2.4 & 1.9 & 3.7 & 3.1 & 2.0 & 5.3 & 2.9 & 2.3 & 5.8 & 2.8 & 2.0 & 4.9 \\
\hline Ribofla & 2.3 & 1.9 & 3.3 & 3.0 & 2.1 & 5.1 & 2.5 & 2.2 & 1.2 & 2.6 & 2.0 & 3.8 \\
\hline Pre-formed niacin (mg) & 26.7 & 24.4 & 11.1 & 28.8 & 25.2 & 13.2 & 27.6 & 24.8 & 11.9 & 27.7 & 24.9 & 12.2 \\
\hline Total niacin equivalents (mg) & 44.7 & 42.3 & 13.3 & 48.0 & 45.1 & 14.7 & 47.7 & 44.4 & 13.6 & 46.7 & 44.0 & 14.0 \\
\hline Vitamin $B_{6}(\mathrm{mg})$ & 4.3 & 2.7 & 9.6 & 5.6 & 2.7 & 11.9 & 4.6 & 3.0 & 8.6 & 4.9 & 2.8 & 10.4 \\
\hline Vitamin $B_{12}(\mu \mathrm{g})$ & 4.8 & 4.0 & 4.1 & 5.9 & 4.4 & 5.1 & 5.9 & 5.0 & 4.4 & 5.5 & 4.4 & 4.6 \\
\hline Folate $(\mu \mathrm{g})$ & 325 & 292 & 144 & 353 & 297 & 185 & 377 & 326 & 258 & 348 & 302 & 192 \\
\hline Biotin ( & 42.9 & 34.1 & 41.8 & 46.9 & 40.8 & 28.1 & 47.2 & 44.0 & 23.4 & 45.5 & 39.1 & 33.1 \\
\hline Pantothenate (mg) & 6.2 & 5.4 & 4.7 & 7.7 & 5.8 & 7.5 & 7.3 & 6.6 & 3.7 & 7.0 & 5.8 & 5.8 \\
\hline Vitamin C (mg) & 134 & 87 & 266 & 150 & 87 & 237 & 169 & 103 & 255 & 148 & 90 & 252 \\
\hline
\end{tabular}

Significantly different from women, $P<0.001$.

b Significantly different from women, $P<0.01$.

Table 17 Percentage of population groups with mean daily vitamin intakes below the average requirement (AR)*

\begin{tabular}{|c|c|c|c|c|c|c|c|c|c|}
\hline & \multirow[b]{2}{*}{$\mathrm{AR}^{\star}$} & \multicolumn{4}{|c|}{ Men } & \multicolumn{4}{|c|}{ Women } \\
\hline & & $\begin{array}{l}18-35 \\
\text { years }\end{array}$ & $\begin{array}{l}36-50 \\
\text { years }\end{array}$ & $\begin{array}{l}51-64 \\
\text { years }\end{array}$ & $\begin{array}{c}\text { All ages } \\
\text { (18-64 years) }\end{array}$ & $\begin{array}{l}18-35 \\
\text { years }\end{array}$ & $\begin{array}{l}36-50 \\
\text { years }\end{array}$ & $\begin{array}{l}51-64 \\
\text { years }\end{array}$ & $\begin{array}{c}\text { All ages } \\
(18-64 \text { years })\end{array}$ \\
\hline Total vitamin A & $500(400) \mu \mathrm{g}$ & 25.3 & 14.4 & 20.8 & 20.2 & 20.1 & 11.9 & 19.1 & 16.6 \\
\hline Thiamin & $0.8(0.6) \mathrm{mg}$ & 1.2 & 1.3 & 0.0 & 0.9 & 1.1 & 0.3 & 0.0 & 0.6 \\
\hline Riboflavin & $1.3(1.1) \mathrm{mg}$ & 13.4 & 12.3 & 11.6 & 12.5 & 26.0 & 17.5 & 16.0 & 20.6 \\
\hline Total niacin equivalents & 15 (11) mg & 0.0 & 0.4 & 0.0 & 0.2 & 0.4 & 0.0 & 0.0 & 0.1 \\
\hline Vitamin $B_{6}$ & $1.3(1.0) \mathrm{mg}$ & 1.6 & 2.1 & 1.2 & 1.7 & 2.2 & 1.4 & 4.3 & 2.4 \\
\hline Vitamin $B_{12}$ & $1.0 \mu \mathrm{g}$ & 0.4 & 0.8 & 0.0 & 0.5 & 1.9 & 0.3 & 0.0 & 0.8 \\
\hline Folate & $140 \mu g$ & 3.2 & 2.5 & 1.2 & 2.4 & 11.2 & 6.6 & 8.6 & 8.8 \\
\hline Vitamin C & $30 \mathrm{mg}$ & 10.3 & 5.9 & 5.8 & 7.6 & 8.9 & 7.7 & 9.9 & 8.6 \\
\hline
\end{tabular}

* Reports of the Scientific Committee for Food, 1993; values in parentheses àre for women.

from liver and $\beta$-carotene from certain vegetables may not be fully captured in a 7-day record of food intake ${ }^{33}$. Misreporting is present in all surveys that use selfreported food intakes and, in particular, underreporting of food intakes will tend to overestimate the percentage of the population with inadequate intakes. Underreporting of energy intake in this survey is addressed elsewhere ${ }^{34}$.

In general, the percentage of the population with mean daily intakes of vitamins below the AR was low, except for vitamin A and riboflavin. The significance of this observation for public health is not clear, as there are no studies on vitamin A or riboflavin status in the 18-64-yearold adult population in Ireland. Evaluation of the adequacy of folate intake in Irish women of reproductive age with respect to the AR of $140 \mu \mathrm{g} \mathrm{day}^{-1}$ (which is established on the basis of maintenance of red blood cell folate levels to prevent deficiency ${ }^{3}$ ) showed that $11.2 \%$ of women aged $18-35$ years and $6.6 \%$ of women aged $36-50$ years had mean daily intakes of folate below the AR. However, the AR for folate does not take into account more recent recommendations for a total intake of about 
Table 18 Percentage of population groups with mean daily vitamin intakes below the lowest threshold intake (LTI)*

\begin{tabular}{|c|c|c|c|c|c|c|c|c|c|}
\hline & \multirow[b]{2}{*}{$\mathrm{LTI}^{*}$} & \multicolumn{4}{|c|}{ Men } & \multicolumn{4}{|c|}{ Women } \\
\hline & & $\begin{array}{l}18-35 \\
\text { years }\end{array}$ & $\begin{array}{l}36-50 \\
\text { years }\end{array}$ & $\begin{array}{l}51-64 \\
\text { years }\end{array}$ & $\begin{array}{c}\text { All ages } \\
\text { (18-64 years) }\end{array}$ & $\begin{array}{l}18-35 \\
\text { years }\end{array}$ & $\begin{array}{l}36-50 \\
\text { years }\end{array}$ & $\begin{array}{l}51-64 \\
\text { years }\end{array}$ & $\begin{array}{c}\text { All ages } \\
\text { (18-64 years) }\end{array}$ \\
\hline Total vitamin A & $300(250) \mu \mathrm{g}$ & 5.9 & 3.4 & 4.0 & 4.5 & 6.3 & 2.4 & 1.9 & 3.8 \\
\hline Vitamin E & $4(3) \mathrm{mg}$ & 11.5 & 15.3 & 24.9 & 16.3 & 7.1 & 10.5 & 17.3 & 10.7 \\
\hline Thiamin & $0.6(0.4) \mathrm{mg}$ & 0.0 & 0.0 & 0.0 & 0.0 & 0.0 & 0.0 & 0.0 & 0.0 \\
\hline Riboflavin & $0.6 \mathrm{mg}$ & 0.4 & 0.4 & 0.0 & 0.3 & 3.0 & 0.0 & 0.6 & 1.3 \\
\hline Total niacin equivalents & $11(9) \mathrm{mg}$ & 0.0 & 0.4 & 0.0 & 0.2 & 0.0 & 0.0 & 0.0 & 0.0 \\
\hline Vitamin $B_{12}$ & $0.6 \mu g$ & 0.0 & 0.8 & 0.0 & 0.3 & 1.1 & 0.0 & 0.0 & 0.4 \\
\hline Folate & $85 \mu \mathrm{g}$ & 0.4 & 0.0 & 0.0 & 0.2 & 1.9 & 0.3 & 0.6 & 1.0 \\
\hline Vitamin C & $12 \mathrm{mg}$ & 0.4 & 0.0 & 0.6 & 0.3 & 0.7 & 0.0 & 0.6 & 0.4 \\
\hline
\end{tabular}

"Reports of the Scientific Committee for Food, 1993; values in parentheses are for women.

$600 \mu \mathrm{g} \mathrm{day}^{-1}$ (i.e. an intake of $400 \mu \mathrm{g}$ day $^{-1}$ from supplements in addition to the estimated $200 \mu \mathrm{g} \mathrm{day}^{-1}$ from food) for women of reproductive age for the prevention of neural tube defects ${ }^{4,5}$. The mean daily folate intakes of women aged 18-35 years and 36-50 years were $247 \mu \mathrm{g}$ and $267 \mu \mathrm{g}$, respectively, and only $2.2 \%$ of women aged $18-35$ years and $5.2 \%$ of women aged 36-50 years achieved an intake of $600 \mu \mathrm{g} \mathrm{day}^{-1}$.

There is no established AR for vitamin $D$ but an acceptable range of intake has been set at 0-10 $\mu \mathrm{g}$, depending on sunlight exposure ${ }^{3}$. The median intakes observed in this survey ( $2.5 \mu \mathrm{g}$ in men, $2.1 \mu \mathrm{g}$ in women) indicate that a considerable proportion of the population has low dietary intake of vitamin D and is largely dependent on sunlight to maintain adequate vitamin $D$ status. It is not clear whether this exposure to sunlight is adequate and there are no data on vitamin $D$ status of 1864-year-old adults in Ireland. However, studies on vitamin D status of adults in other European countries suggest that a significant prevalence of low vitamin D status exists ${ }^{35-37}$. The contribution of vitamin D from spreading fats $(\approx 5 \%)$ is lower than that estimated in the Irish National Nutrition Survey ${ }^{19}(24-39 \%)$ and it is noted that vitamin $\mathrm{D}$ is added to only a minority of spreading fats on sale in Ireland.

The criteria of adequacy used for establishing the AR do not take into consideration other possible health benefits of an increased intake of certain vitamins beyond current recommendations, e.g. the reduction of plasma homocysteine and the risk of coronary heart disease by folate ${ }^{6}$ or the reduction of the risk of certain cancers by vitamin $\mathrm{C}^{7}$. However, although there is a growing body of evidence to support such benefits, they have yet to be confirmed.

The lowest threshold intake (LTI) for a nutrient (the intake level below which nearly all individuals will be unable to maintain metabolic integrity according to the criterion used for each nutrient ${ }^{3}$ ) is sometimes used as a cut-off value to assess the prevalence of nutrient inadequacy. However, while the LTI can be used to detect individuals with a very high probability of inadequate intakes, it is of limited value for assessing the prevalence of nutrient inadequacy in populations. For example, $3.8 \%$ of women had vitamin $\mathrm{A}$ intakes less than the LTI of $250 \mu \mathrm{g}$ (Table 18) but the estimate of vitamin A inadequacy in this population group was $16.6 \%$ using the $\mathrm{AR}$ as a cut-off value (Table 17). For vitamin $\mathrm{E}$, for which no AR has been established (since recommended intakes are related to polyunsaturated fatty acid (PUFA) intake), the proportion of men (16.3\%) and women (10.7\%) with mean daily intakes less than the LTI ( $4 \mathrm{mg}$ for men and $3 \mathrm{mg}$ for women, regardless of PUFA intake) indicates that the prevalence of vitamin $\mathrm{E}$ inadequacy may be significant.

The tolerable upper intake level (UL) is the highest level of daily nutrient intake that is unlikely to pose risk of adverse health effects in almost all individuals in the specified life-stage group ${ }^{8}$. ULs for adults have been established for retinol $(3000 \mu \mathrm{g})^{11}$, vitamin $D(50 \mu \mathrm{g})^{8}$, vitamin $\mathrm{E}(1000 \mathrm{mg})^{10}$, vitamin $\mathrm{B}_{6}(25 \mathrm{mg})^{14}$, folic acid $(1000 \mu \mathrm{g})^{16}$ and vitamin $\mathrm{C}(2000 \mathrm{mg})^{10}$. No ULs have been set for thiamin ${ }^{9}$, riboflavin ${ }^{13}$, vitamin $\mathrm{B}_{12}{ }^{15}$, biotin ${ }^{9}$ and pantothenate ${ }^{9}$ due to lack of evidence of adverse effects, while no UL has been set for $\beta$-carotene due to inconsistency in evidence of possible adverse effects ${ }^{12}$. Except for pre-formed niacin, the 95th percentile intakes of vitamins did not exceed the UL for any group and were much lower than the UL for most vitamins. The 95th percentile intake of retinol for men aged 51-64 years $\left(2784 \mu \mathrm{g} \mathrm{day}^{-1}\right.$ ) approached the UL for retinol ${ }^{11}$ of $3000 \mu \mathrm{g} \mathrm{day}^{-1}$, with $4.0 \%$ of men in this age group exceeding the UL. The 95th percentile for retinol intake for women aged 51-64 years (2354 $\mathrm{g} \mathrm{day}^{-1}$ ) was lower than the UL, with $1.2 \%$ of women in this age group having mean daily retinol intakes that exceeded the UL. The 95th percentile for retinol intakes for women in the 18-35 year and 36-50 year age groups was well below the UL. Also, $1.5 \%$ of women aged $18-35$ years and $2.4 \%$ of women aged $36-50$ years had mean daily intakes that exceeded the UL, suggesting that there is low risk of retinol toxicity in the population.

The 95th percentile intakes of pre-formed niacin from all sources for all groups exceeded the $\mathrm{UL}^{9}$ of $35 \mathrm{mg}$, and $20.8 \%$ of men of all ages and $6.3 \%$ of women of all ages had mean 
daily intakes greater than the UL. The UL for niacin is set on the basis of the occurrence of flushing of the skin in response to nicotinic acid, a transient effect ${ }^{9}$. However, over $40 \%$ of the intake of pre-formed niacin is obtained from meat, meat products and fish, where it is present largely as nicotinamide $^{38}$ which does not appear to cause flushing ${ }^{9}$. Thus, the risk of overexposure to nicotinic acid is likely to be considerably overestimated by this analysis and probably is related solely to supplement use ${ }^{39}$. The 95th percentile intake of vitamin $\mathrm{B}_{6}$ was well below the $\mathrm{UL}^{11}$ of $25 \mathrm{mg}$, and $0.2 \%$ of men and $2.0 \%$ of women had intakes that exceeded the UL. Of the 14 women whose intakes of vitamin $\mathrm{B}_{6}$ exceeded the UL, the median intake was $43 \mathrm{mg} \mathrm{day}^{-1}$ and all were supplement users ${ }^{39}$. It is noted that the 95 th percentile intake of folate was well below the UL for folic acid, $1000 \mu \mathrm{g}$ for all age group ${ }^{16}$, and that mean daily folate intakes were above the UL in $0.2 \%$ of men and $0.3 \%$ of women, indicating that there is little risk of adverse effects in the population from current levels of dietary folate. Similarly, for vitamin C, it was noted that the 95 th percentile intake was well below the UL for vitamin C of $2000 \mathrm{mg}$ for all age groups ${ }^{10}$ and the mean daily vitamin $C$ intake was above the UL in $0.2 \%$ of men and $0.3 \%$ of women. This again indicates that there is little risk of adverse effects in the population from current levels of dietary vitamin $\mathrm{C}$.

The lower mean nutrient density of vitamin intakes for men than women for most vitamins may reflect dietary patterns in men, which are relatively richer in potatoes and alcoholic beverages and relatively poor in fruits, vegetables, breakfast cereals and nutritional supplements. Similarly, the differences in nutrient densities of vitamin intakes between age groups for both men and women may reflect differences in consumption of particular food groups, such as meat, milk, fruits and vegetables, potatoes and potato products, breakfast cereals and alcoholic beverages, as well as different patterns of supplement use. Further analysis of the database will be carried out to explain the age and sex differences in nutrient density.

Dairy products made a significant contribution to dietary intakes of vitamin $A$, vitamin $B_{12}$, riboflavin and pantothenate; meat and meat products to vitamins $A$ and $\mathrm{D}$, thiamin, riboflavin, niacin, vitamin $\mathrm{B}_{12}$, vitamin $\mathrm{B}_{6}$, pantothenate and biotin; vegetables and vegetable dishes to carotene, vitamin A, vitamin $\mathrm{E}$, folate and vitamin $\mathrm{C}$; potato and potato products to vitamin $\mathrm{A}$, vitamin $\mathrm{E}$, thiamin, pantothenate, vitamin $B_{6}$, folate and vitamin $C$; fish and fish products to vitamin $D$ and vitamin $B_{12}$; eggs and egg dishes to vitamin $D$; fruit and fruit juices to vitamin $\mathrm{C}$; bread and rolls to thiamin, niacin and folate; and breakfast cereals to thiamin, riboflavin and folate. The primary dietary sources of vitamin $\mathrm{E}$ are vegetable oils and wholegrains ${ }^{40}$. These sources are incorporated within the food groups vegetables \& vegetable dishes, potatoes \& potato products, bread \& rolls and breakfast cereals. Overall, the contribution of nutritional supplements to the mean daily intakes of men and women of all ages for any vitamin was less than $12 \%$. However, supplements did contribute quite significantly to the mean daily intakes of some vitamins in supplement consumers ${ }^{39}$.

Direct comparison of vitamin intakes in the present survey with those estimated in earlier surveys in Ireland is difficult, due to the different methods used for food intake estimation. However, comparison of vitamin intakes per $10 \mathrm{MJ}$ energy shows some important differences. Nutrient density in the present survey was higher for both men and women for most vitamins when compared with the Irish National Nutrition Survey $(\mathrm{INNS})^{19}$. In particular, the nutrient densities for folate, vitamin $\mathrm{C}$ and vitamin $\mathrm{E}$ were substantially higher than estimated in the INNS $^{19}$. The difference in nutrient density can in part be explained by the inclusion of nutritional supplements in the present survey, unlike the INNS survey. However, nutritional supplements contributed a maximum of $12 \%$ to the intake of any vitamin and a maximum of $4.6 \%, 7.1 \%$ and $8.8 \%$ respectively to folate, vitamin $C$ and vitamin $E$ for any population group, indicating that the apparent increase in nutrient density is only partly due to supplement use. Given the significant changes in the food supply in Ireland over the past 10 years it is likely that an increase in vitamin intakes from certain foods may have occurred, e.g. vitamin $\mathrm{C}$ and folic acid and other $\mathrm{B}$ vitamins from fortified foods.

\section{Conclusions}

The percentage of the population with mean daily intakes below the AR was low except for vitamin A and riboflavin. While no AR was available for vitamin $\mathrm{E}$, the numbers of individuals with vitamin $\mathrm{E}$ intakes below the LTI indicate a significant level of inadequacy of this vitamin in this population group. Folate intakes in women of reproductive age are not meeting current recommendations for preventing neural tube defects. A high proportion of the population had a low dietary intake of vitamin $D$ and is largely dependent on sunlight exposure to maintain adequate vitamin $D$ status. With the possible exception of niacin (flushing) and vitamin $\mathrm{B}_{6}$ (neuropathy), there appears to be little risk of the occurrence of adverse effects due to excessive consumption of vitamins in this population, based on current dietary practices.

\section{References}

1 Food Safety Authority of Ireland. Recommended Dietary Allowances for Ireland. Dublin: Government Publications, 1999.

2 Department of Health. Dietary Reference Values for Food Energy and Nutrients for the United Kingdom. London: HMSO, 1991.

3 Scientific Committee for Food. Nutrient and Energy Intakes for the European Community. Luxembourg: Office for Official Publications of the European Communities, 1993.

4 Food Safety Advisory Board. The Value of Folic Acid in the Prevention of Neural Tube Defects. Dublin: Government Publications, 1998. 
5 Department of Health. Folic Acid and the Prevention of Disease. London: HMSO, 2000.

6 Schorah CJ, Devitt H, Lucock M, Dowell AC. The responsiveness of plasma homocysteine to small increases in dietary folic acid: a primary care study. Eur. J. Clin. Nutr. 1998; 52: 407-11.

7 Department of Health. Nutritional Aspects of the Development of Cancer. London: HMSO, 1998.

8 Food and Nutrition Board, Institute of Medicine. Dietary Reference Intakes: Calcium, Phosphorous, Magnesium, Vitamin $D$ and Fluoride. Washington, DC: National Academy Press, 1997.

9 Food and Nutrition Board, Institute of Medicine. Dietary Reference Intakes: Thiamin, Riboflavin, Niacin, Vitamin $B_{6}$, Vitamin $B_{12}$, Pantothenic Acid, Biotin, Folate and Choline. Washington, DC: National Academy Press, 1998.

10 Food and Nutrition Board, Institute of Medicine. Dietary Reference Intakes for Vitamin C, Vitamin E, Selenium and Carotenoids. Washington, DC: National Academy Press, 2000.

11 Food and Nutrition Board, Institute of Medicine. Dietary Reference Intakes: Vitamin A, Vitamin K, Arsenic, Boron, Chromium, Copper, Iodine, Iron, Manganese, Molybdenum, Nickel, Silicon, Vanadium and Zinc. Washington, DC: National Academy Press, 2001 [prepublication copy].

12 Scientific Committee for Food. Opinion of the Scientific Committee for Food on the Tolerable Upper Intake Level of $\beta$-Carotene [Online]. Available at http://www.europa. eu.int. 19 October 2000.

13 Scientific Committee for Food. Opinion of the Scientific Committee for Food on the Tolerable Upper Intake level of Riboflavin [Online]. Available at http://www.europa.eu.int. 22 November 2000.

14 Scientific Committee for Food. Opinion of the Scientific Committee for Food on the Tolerable Upper Intake level of Vitamin $B_{6}$ [Online]. Available at http://www.europa.eu.int. 19 October 2000.

15 Scientific Committee for Food. Opinion of the Scientific Committee for Food on the Tolerable Upper Intake level of Vitamin $B_{12}$ [Online]. Available at http://www.europa. eu.int. 19 October 2000.

16 Scientific Committee for Food. Opinion of the Scientific Committee for Food on the Tolerable Upper Intake level of Folate [Online]. Available at http://www.europa.eu.int. 19 October 2000.

17 Scientific Co-operation Task 7.1.1 Working Group. Scientific Considerations for the Development of Measures on the Addition of Vitamins and Minerals to Foodstuffs. Luxembourg: Office for Official Publications of the European Communities, 1997.

18 Brussard JH, Russ D, Fletcher R, Moreiras O, van den Berg H. Paper 1: Nutrient Intakes and Status in Europe. Belgium: International Life Sciences Institute, 1998.

19 Lee P, Cunningham K. The Irish National Nutrition Survey 1990. Dublin: The Irish Nutrition and Dietetic Institute, 1990.

20 Harrington $\mathrm{KE}$, Robson PJ, Kiely $\mathrm{M}$, Livingstone MBE, Lambe J, Gibney MJ. The North/South Ireland Food Consumption Survey: survey design and methodology. Public Health Nutr. 2001; 4(5A): 1037-42.

21 Kiely M, Flynn A, Harrington KE, Robson PJ, Cran G. Sampling description and procedures used to conduct the North/South Ireland Food Consumption Survey. Public Health Nutr. 2001; 4(5A): 1029-35.

22 Holland B, Welch AA, Unwin ID, Buss DH, Paul AA, Southgate DAT. McCance $\mathcal{E}$ Widdowson's The Composition of Foods, 5th ed. Royal Society of Chemistry and Ministry of Agriculture, Fisheries and Food. London: HMSO, 1995.

23 Holland B, Unwin ID, Buss DH. Cereals and Cereal Products. Third Supplement to McCance \& Widdowson's The Composition of Foods, 4th ed. Royal Society of
Chemistry and Ministry of Agriculture, Fisheries and Food. London: HMSO, 1988.

24 Holland B, Unwin ID, Buss DH. Milk Products and Eggs. Fourth Supplement to McCance \& Widdowson's The Composition of Foods, 4th ed. Royal Society of Chemistry and Ministry of Agriculture, Fisheries and Food. London: HMSO, 1989.

25 Holland B, Unwin ID, Buss DH. Vegetables, Herbs and Spices. Fifth Supplement to McCance $\mathcal{E}$ Widdowson's The Composition of Foods, 4th ed. Royal Society of Chemistry and Ministry of Agriculture, Fisheries and Food. London: HMSO, 1991.

26 Holland B, Unwin ID, Buss DH. Fruit and Nuts. First Supplement to McCance \& Widdowson's The Composition of Foods, 5th ed. Royal Society of Chemistry and Ministry of Agriculture, Fisheries and Food. London: HMSO, 1992.

27 Holland B, Welch AA, Buss DH. Vegetable Dishes. Second Supplement to McCance E Widdowson's The Composition of Foods, 5th ed. Royal Society of Chemistry and Ministry of Agriculture, Fisheries and Food. London: HMSO, 1996.

28 Holland B, Brown J, Buss DH. Fish and Fish Products. Third Supplement to McCance $\mathcal{E}$ Widdowson's The Composition of Foods, 5th ed. Royal Society of Chemistry and Ministry of Agriculture, Fisheries and Food. London: HMSO, 1993.

29 Chan W, Brown J, Buss DH. Miscellaneous Foods. Fourth Supplement to McCance E Widdowson's The Composition of Foods, 5th ed. Royal Society of Chemistry and Ministry of Agriculture, Fisheries and Food. London: HMSO, 1994.

30 Chan W, Brown J, Lee SM, Buss DH. Meat, Poultry and Game. Supplement to McCance \& Widdowson's The Composition of Foods. Royal Society of Chemistry and Ministry of Agriculture, Fisheries and Food. London: HMSO, 1995.

31 Chan W, Brown J, Church SM, Buss DH. Meat Products and Dishes. Supplement to McCance $\&$ Widdowson's The Composition of Foods. Royal Society of Chemistry and Ministry of Agriculture, Fisheries and Food. London: HMSO, 1996.

32 Carriquiry AL. Assessing the prevalence of nutrient inadequacy. Public Health Nutr. 1999; 2(1): 23-33.

33 Bingham SA, Gill C, Welch A, Day K, Cassidy A, Khaw KT, Sneyd MJ, Key TJA, Roe L, Day NE. Comparison of dietary assessment methods in nutritional epidemiology: weighed records v. $24 \mathrm{~h}$ recalls, food-frequency questionnaires and estimated-diet records. Br. J Nutr. 1994; 72: 619-43.

34 McGowan $\mathrm{MJ}$, Harrington $\mathrm{KE}$, Kiely $M$, Robson $\mathrm{PJ}$, Livingston MBE, Gibney MJ. An evaluation of energy intakes and the ratio of energy intake to estimated basal metabolic rate (EI/BMR est $)$ in the North/South Ireland Food Consumption Survey. Public Health Nutr. 2001; 4(5A): 1043-50.

35 McKenna MJ. Differences in vitamin D status between countries in young adults and the elderly. Am.J. Med. 1992; 93(1): 69-77.

36 Chapuy MC, Preziosi P, Maamer M, Arnaud S, Galan P, Hereberg S, Meunier PJ. Prevalence of vitamin D insufficiency in an adult normal population. Osteoporosis Int. 1997; 7(5): 439-43.

37 Finch S, Doyle W, Lowe C, Bates CJ, Prentice A, Smithers G, Clarke PC. National Diet and Nutrition Survey: People aged 65 years and over. Volume 1. Report of the Diet and Nutrition Survey. London: HMSO, 1998.

38 Groff JL, Gropper SS, Hunt SM. Advanced Nutrition and Human Metabolism, 2nd ed. St Paul, MN: West Publishing Company, 1995.

39 Kiely M, Flynn A, Harrington KE, Robson PJ, O'Connor N, Hannon EM, O'Brien MM, Bell S, Strain JJ. The efficacy and safety of nutritional supplement use in a representative sample of adults in the North/South Ireland Food Consumption Survey. Public Health Nutr. 2001; 4(5A): 1089-97.

40 Machlin LJ. Handbook of Vitamins: Nutritional, Biocbemical and Clinical Aspects. New York: Dekker, 1984. 